\title{
Electron Tomography of Microelectronic Device Interconnects
}

\author{
Qing Yang $^{*}$, John Mardinly ${ }^{* *}$, Christian Kübel $^{* * *}$, Chris Nelson $^{* * * *}$, and Christian Kisielowski ${ }^{* * * *}$ \\ *Dept. of Materials Science and Engineering, University of California, Berkeley, CA 94720, USA \\ *** Intel Corporation, 2200 Mission College Blvd., SC9-7, Santa Clara, CA 95054, USA \\ ***FEI Company, Eindhoven, Netherlands \\ ${ }^{* * * *}$ NCEM, Lawrence Berkeley National Laboratory, Berkeley, CA 94720, USA
}

The continual shrinking of microelectronic devices and structures governed by Moore's law has resulted in commercial products today incorporating complex non-planar structures having dimensions comparable to or smaller than the thickness of a TEM specimen. As a result, conventional cross-sectioning followed by TEM-STEM imaging frequently yields unsatisfactory results that are difficult to interpret due to geometrical blurring. This situation will become more significant as yet smaller structures in development today become commercial products in coming years. Electron tomography has long been viewed as one of the more promising solutions to this conundrum. In this paper we present results on 3D reconstructions from tilt series, both in bright field TEM and HAADF-STEM images. The samples studied are vias imbedded in $\mathrm{SiO}_{2}$ dielectrics and coated with $\mathrm{Ta} / \mathrm{TaN}$ barrier and $\mathrm{Cu}$ seed layers. Specimens were prepared using Focused Ion Beam in-situ lift-out.

The fidelity of reconstructions is limited by missing data wedges in Fourier space due to incomplete tilting [1]. Combining tilt series from two orthogonal axes [2,3] has been proposed to improve the fidelity of reconstructions through partial reduction of the missing data from wedge to pyramid. Fig. 1 shows a comparison between slices of two single-axis tomograms and a dual-axis tomogram combining the two single-axis tilt series, acquired in a CM30 Ultra-twin using bright field imaging conditions, and reconstructed using IMOD [4], which requires gold markers. The first tilt series (a) consists of 46 images collected from $-40^{\circ}$ to $+50^{\circ}$, and the second tilt series (b) with tilt axis perpendicular to the first one, consists of 57 images collected from $-50^{\circ}$ to $+64^{\circ}$, both at $2^{\circ}$ intervals. Figs. $3 \mathrm{~d}$-f show slices perpendicular to the via with missing sections of the via side wall near the top and bottom surfaces of the TEM membrane. Comparing the reconstruction from single- and dualaxis tilt series, we do not observe a substantial reduction of the missing via side wall. Under our experimental condition, i.e. moderate tilt angle range, the improvement brought by dual-axis tilt is not satisfactory considering the amount of effort required. Moreover, the extended time under the $300 \mathrm{KV}$ beam led to radiation damage of the structures.

Fig. 2 shows the slices from the tomogram reconstructed using images of a different via on the same wafer acquired in a Tecnai F20 using STEM-HAADF conditions at 200KV. The tomogram was reconstructed from 155 images acquired around a single tilt axis over a range of $\pm 75^{\circ}$ at $1^{\circ}$ intervals using marker-less commercial software from FEI. The Ta barrier and $\mathrm{Cu}$ seed layers can be clearly distinguished. Slices perpendicular to the via (Fig. 4b) show that the missing part of the via side wall is substantially reduced (Fig. 4 b) as a result of the increased tilt angle range, and radiation damage is not an issue.

In conclusion, tomograms reconstructed using tilt series of BF TEM and STEM HAADF images both clearly reveal 3D features of the via. Because significant high tilt angle data is missing, part of 
the via wall is blurred and/or missing. Dual-axis tilt tomography with only a moderate tilt-angle range does not effectively improve the fidelity of the reconstruction. In contrast, the fidelity of the reconstruction is greatly improved using single-axis tomography with a large tilt-angle range of $\pm 75^{\circ}$. This work suggests that a single-axis tilt series of STEM HAADF images with a high $\left(> \pm 70^{\circ}\right)$ tiltangle range and a reconstruction software package with an accurate marker-less alignment method are desirable for electron tomography for of microelectronic device structures.

\section{References}

[1] J. Carazo, Fidelity of 3D reconstructions, in: J. Frank (Ed.), Electron Tomography: Threedimensional Imaging with the transmission Electron Microscope, Plenum Press, New York, London, 1992.

[2] P. Penczek, M. Marko, K. Buttle, and J. Frank, Ultramicroscopy 60, 393-410 (1995).

[3] D. N. Mastronarde, J. Struct. Biol. 120, 343-352 (1997).

[4] J. R. Kremer, D.N. Mastronarde, and J.R. McIntosh, J. Struct. Biol. 116, 71-76 (1996). [5] This work is partially supported by the Director, Office of Science, Office of Basic Energy Sciences, of the U.S. Department of Energy under Contract No. DE-AC03-76SF00098.
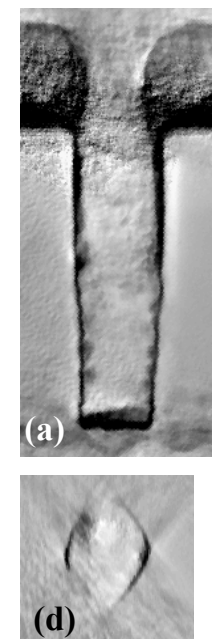
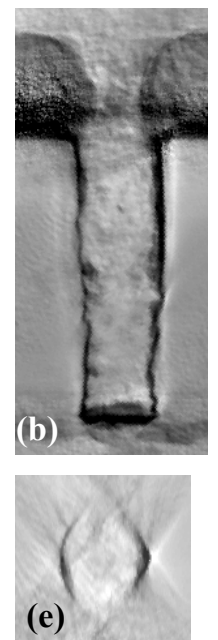

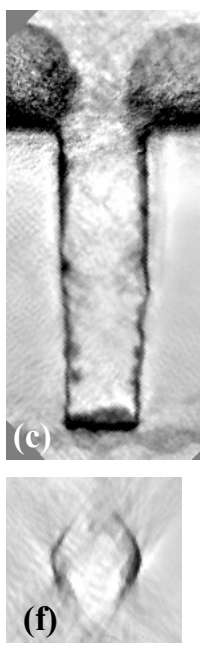

Fig. 1. Slices from tomograms of a cylindrical via reconstructed from BF TEM images. Slices are approximately $2 \mathrm{~nm}$ thick. Panels on the left and in the middle are from tomograms reconstructed from two single-axis tilt series. Panels on the right (c), (f) are from the combined tomogram.
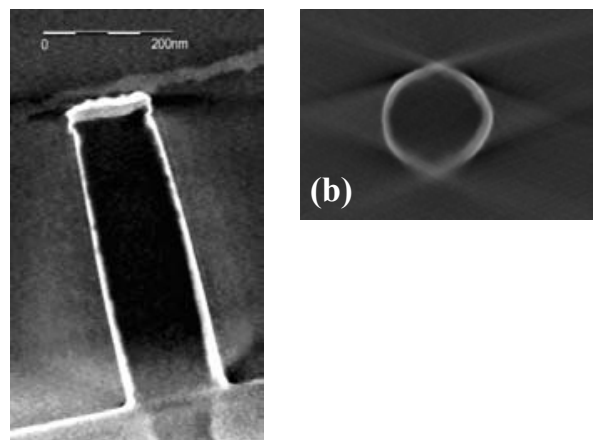

(a)
Fig. 2. Slices from tomogram reconstructed using 155 STEM-HAADF images tilted around one axis at $1^{\circ}$ intervals. (a) Y-Z slice parallel to the TEM sample surface. (b) Slice perpendicular to the sample surface and the long axis of the via. 